\title{
Impaired T lymphocyte function increases tumorigenicity and decreases tumor latency in a mouse model of head and neck cancer
}

\author{
TONY K.S. KU and DAVID L. CROWE \\ University of Illinois Cancer Center, Chicago, IL 60612, USA \\ Received June 15, 2009; Accepted August 10, 2009
}

DOI: 10.3892/ijo_00000438

\begin{abstract}
Squamous cell carcinoma of the head and neck (HNSCC) is the sixth most frequent cancer worldwide. SCC is the most common malignant tumor of the oral cavity with over 35,000 cases and 8,000 deaths reported in the United States each year. Previous case studies have reported increased incidence of HNSCC in patients on immunosuppressive therapy for organ transplantation. The results of these studies indicate that effective immune surveillance is important for preventing emergence of HNSCC. HNSCC may also inhibit immune response to tumor cells, which may be responsible for progression. We previously reported induction of metastatic HNSCC in p53 null mutant mice. Despite induction with the potent carcinogen dimethylbenzanthracene, each mouse developed only 1-2 primary tumors with a relatively long induction period of 22 weeks. We hypothesized that immune surveillance might eliminate early tumor cells resulting in the relatively small number of primary tumors and long induction time. To test this hypothesis we performed the induction protocol in nude mice which have defective $\mathrm{T}$ lymphocyte function. Decreased T lymphocyte function resulted in reduced tumor latency and increased tumor formation. Immunohistochemical studies showed that expression of cell cycle regulatory proteins is similar in mouse and human HNSCC. However, distinct differences exist between primary and metastatic tumors from nude and wild-type mice. We also determined that lymphocytes react to metastatic tumor cells by upregulating immunoglobin gene expression but are prone to apoptosis via decreased expression of survival factors and upregulation of cell death genes.
\end{abstract}

\section{Introduction}

Squamous cell carcinoma of the head and neck (HNSCC) is the sixth most frequent cancer worldwide (1). HNSCC is a major cause of morbidity and mortality in developing

Correspondence to: Dr David L. Crowe, University of Illinois Cancer Center, 801 S. Paulina Street, Room 530C, Chicago, IL 60612, USA

E-mail: dlcrowe@uic.edu

Key words: squamous cell carcinoma, immunology, carcinogenesis, antibody, metastasis nations, comprising up to $50 \%$ of all malignant tumors. SCC is the most common malignant tumor of the oral cavity with over 35,000 cases and 8,000 deaths reported in the United States each year (2). Tobacco carcinogens are the primary etiologic agents of the disease with age and genetic background as contributory factors. The overall 5-year survival rate is low among the major cancers and has not declined significantly in recent years.

Previous case studies have reported increased incidence of HNSCC in patients on immunosuppressive therapy for organ transplantation (3-7). Induction of HNSCC was second to that of liver cancer in patients with bone marrow transplant (8). The results of these studies indicate that effective immune surveillance is important for preventing emergence of HNSCC. HNSCC may also inhibit immune response to tumor cells, which may be responsible for progression (9). It has been proposed that immune surveillance consists of three phases: elimination, equilibrium, and escape $(10,11)$. Tumor elimination is the goal of immunosurveillance, but during equilibrium and escape the interactions between the immune system and tumor cells may result in development of cells which can gradually inactivate or kill immune effector cells. Later stages of tumor development may include cells which are less immunogenic. Natural killer and cytotoxic T lymphocytes which have crucial effector functions in immune defense against tumor cells are inactivated in $\operatorname{HNSCC}(12,13)$. Regression of tumor grafts is characterized by NK and cytotoxic $\mathrm{T}$ lymphocyte attack, and patients with metastatic HNSCC have low NK and cytotoxic T lymphocyte activity $(14,15)$. Spontaneous apoptosis of circulating T lymphocyte populations in patients with HNSCC is an indicator of diminished immune function (16). Cytokine induced stimulation of the immune system and transfer of effector cells have not been effective in head and neck cancer therapy $(17,18)$. Tumor infiltrating lymphocytes are not cytotoxic to autologous tumor cells and exhibit reduced clonogenicity (19). Human HNSCC have been shown to induce minimal cell mediated anti-tumor immune responses (20). Diminished numbers of cytotoxic T lymphocytes correlated with reduced survival in cancer patients (21). A variety of cellular defects such as signaling abnormalities, spontaneous apoptosis, and reduced proliferation have been reported in natural killer, T lymphocyte, and dendritic cells in HNSCC patients $(16,22)$. The results of these studies indicate that cell mediated immune responses are critically important in tumor initiation and progression. 
We previously reported induction of metastatic HNSCC in p53 null mutant mice (23). These tumors ranged from well to poorly differentiated histopathology and showed many molecular features of human HNSCC. These tumors progressed rapidly which resulted in euthanasia criteria being met in 13 weeks. Despite induction with the potent carcinogen dimethylbenzanthracene, each mouse developed only 1-2 primary tumors with a relatively long induction period of 22 weeks. We hypothesized that immune surveillance might eliminate early tumor cells resulting in the relatively small number of primary tumors and long induction time. To test this hypothesis we performed the induction protocol in nude mice which have defective $\mathrm{T}$ lymphocyte function. Our results show that defective $\mathrm{T}$ lymphocyte function reduces tumor latency period and significantly increases tumor number. Global gene expression profiling demonstrated important differences between HNSCC induced in nude and wild-type mice. We also profiled gene expression in lymphocytes exposed to metastatic tumor cells in vivo. Tumor cell exposed lymphocytes upregulate immunoglobin gene expression but are prone to apoptosis via decreased expression of survival factors and upregulation of cell death genes. The results of our study indicate that $\mathrm{T}$ lymphocyte function is an important regulator of tumor development in HNSCC.

\section{Materials and methods}

Mouse procedures. This study was approved by the Institutional Animal Care and Use Committee before any experiments were performed. The Foxn1 mutant (nude) mouse strain was purchased from The Jackson Laboratory (Bar Harbor, ME). C57B16J mice were used as control animals. Mice were housed in approved environmentally controlled facilities on 12-h light-dark cycles with unlimited access to food and water. Twenty male and female 1-month old nude and wild-type mice were dosed orally twice weekly using $25 \mu 1$ dimethylbenzanthracene (DMBA) dissolved in $20 \mu 1$ ethanol. The time course and number of tumors were recorded for each animal. Mice were euthanized when any institutional criterion for experimental neoplasia in rodents was met. Complete necropsies were performed on each mouse. A portion of each tumor specimen was flash frozen in liquid nitrogen or fixed in $4 \%$ buffered formaldehyde for $16 \mathrm{~h}$ at room temperature.

Histopathology and immunohistochemistry. Tumor tissue was dehydrated in an ethanol series, cleared in xylene, and embedded in paraffin. Five micrometer sections were prepared and mounted on poly-L-lysine coated slides. Representative sections were stained with hematoxylin and eosin and histologically evaluated by a pathologist. Immunohistochemical analysis was performed using a commercially available kit (Invitrogen, Carlsbad, CA). Sections were incubated at $60^{\circ} \mathrm{C}$ for $30 \mathrm{~min}$ and deparaffinized in xylene. Endogenous peroxidase activity was quenched by incubation in a 9:1 methanol/30\% hydrogen peroxide solution for $10 \mathrm{~min}$ at room temperature. Sections were rehydrated in PBS ( $\mathrm{pH} 7.4)$ for $10 \mathrm{~min}$ at room temperature. Sections were blocked with $10 \%$ normal serum for $10 \mathrm{~min}$ at room temperature followed by incubation with anti-EGFR, cyclin A, cyclin B, cyclin D, cyclin E, p16, c-myc, HGF, TGF $\alpha$, c-met, and PCNA antibodies (Santa Cruz Biotechnology, Santa Cruz, CA) for $16 \mathrm{~h}$ at room temperature.
After three washes in PBS, the sections were incubated with secondary antibody conjugated to biotin for $10 \mathrm{~min}$ at room temperature. After additional washes in PBS, the sections were incubated with streptavidin conjugated horseradish peroxidase for $10 \mathrm{~min}$ at room temperature. Following final washes in PBS, antigen-antibody complexes were detected by incubation with hydrogen peroxide substrate solution containing aminoethylcarbazole chromogen reagent. Slides were rinsed in distilled water, coverslipped using aqueous mounting medium, and allowed to dry at room temperature. The relative intensities of the completed immunohistochemical reactions were evaluated using light microscopy by independent trained observers who were unaware of the mouse genotypes. A scale of 0-3 was used to score relative intensity, with 0 corresponding to no detectable immunoreactivity and 1,2, and 3 equivalent to low, moderate, and high expression respectively. Non-parametric data was analyzed by Fisher exact test.

RNA extraction and gene expression profiling. Total RNA was extracted from microdissected primary and metastatic tumor tissue using a commercially available kit (RNasy, Qiagen, Valencia, CA). Individually matched well differentiated primary and metastatic tumor tissue was used in microarray analysis. Three independent samples from each group were used in gene expression analysis. The integrity of rRNA bands was confirmed by Northern gel electrophoresis. Total RNA $(10 \mu \mathrm{g})$ with spike in controls was reverse transcribed using a T7-oligo(dT) promoter primer in the first strand cDNA synthesis reaction. Following RNase $\mathrm{H}$ mediated second strand synthesis, the double stranded cDNA was purified and served as template in the subsequent in vitro transcription reaction. The in vitro transcription reaction was carried out in the presence of T7 RNA polymerase and a biotinylated nucleotide analogue/ribonucleotide mix for complementary RNA amplification and biotin labeling. The biotinylated complementary RNA targets were then purified, fragmented, and hybridized to Affymetrix GeneChip Expression arrays (Santa Clara, CA). The murine genome 4302.0 microarray was used to interrogate 39,000 possible transcripts in each sample. After washing, hybridization signals were detected using streptavidin conjugated phycoerythrin. Affymetrix GCOS software was used to generate raw gene expression scores and normalized to the relative hybridization signal from each experiment. All gene expression scores were set to a minimum value of 2 times background determined by GCOS software in order to minimize noise associated with less robust measurements of rare transcripts. Normalized gene expression data was imported into dChip sotware for hierarchical clustering analysis using the average linkage algorithm. Raw data was analyzed for quality control and the significance of differential gene expression determined by t-test $(\mathrm{p}<0.05)$ and ratio analysis (>2-fold).

\section{Results}

We used the chemical carcinogenesis protocol described in Materials and methods to induce head and neck cancer in the nude mouse. As shown in Fig. 1, nude mice developed tumors by 14 weeks induction on average compared to 22 weeks for wild-type animals $(\mathrm{p}<0.0002)$. This latency period was 4 weeks 


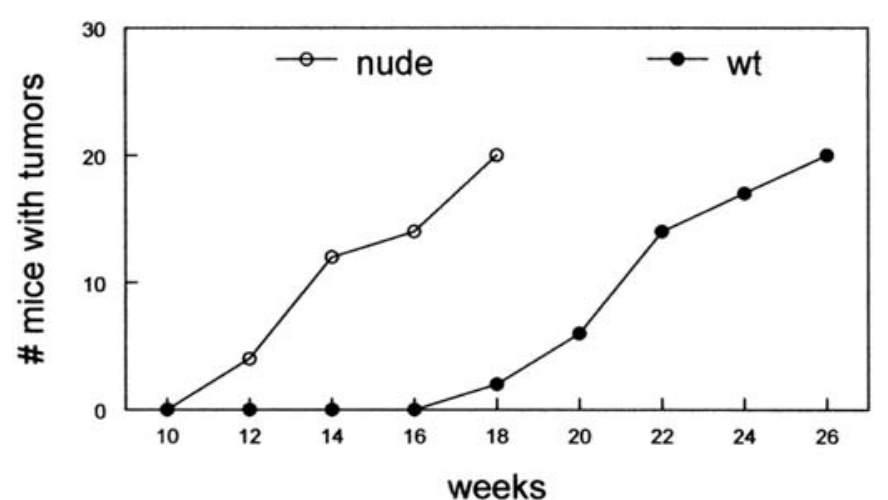

Figure 1. Decreased tumor latency in a nude mouse model of head and neck cancer. Squamous cell carcinoma was induced as described in Materials and methods. The total number of mice which developed tumors each week was recorded.

shorter than tumor formation in $\mathrm{p} 53+/-$ mice as previously reported (23). There also was a dramatic increase in the number of tumors in nude mice (Fig. 2A). Nude mice developed 5.1 tumors per mouse while wild-type animals had only 1.4 lesions/mouse $\left(\mathrm{p}<10^{-12}\right)$. There were no significant differences in primary tumor growth rates or number of metastatic lymph nodes in nude and wild-type mice (Fig. 2B and $\mathrm{C}$ ). These results indicate that decreased $\mathrm{T}$ lymphocyte function results in reduced tumor latency and increased tumor formation.

We examined the histopathology of head and neck tumors in both nude and wild-type mice. In nude mice, primary tumors were well differentiated SCC which developed primarily on labial and buccal mucosa (Fig. 3A). In wild-type mice, $60 \%$ of SCC were well differentiated, $30 \%$ were moderately differentiated, and $10 \%$ were poorly differentiated as previously reported (23). We examined expression of several cell cycle regulatory proteins in tissue sections of primary tumors by immunohistochemistry. We previously demonstrated correlations between expression of these proteins in human HNSCC (24). Epidermal growth factor receptor, PCNA, cyclin A, cyclin B, cyclin D, cyclin E, HGF, c-met, and TGF $\alpha$ were overexpressed in $40-50 \%$ of SCC in nude mice (Fig. 3B-H and J-L). c-myc was overexpressed in $25 \%$ of primary SCC (Fig. 3I). These percentages were similar to those observed in wild-type mice. These results indicate that expression of cell cycle regulatory proteins is similar in SCC in nude and wild-type mice.

The average number of cervical lymph nodes containing metastatic tumor cells was 4.1 per mouse, which was not significantly different between nude and wild-type mice. At necropsy, there was no evidence of tumor extension through the lymph node capsule or distant metastasis to any organ. Metastatic tumor in cervical lymph nodes were uniformly moderately differentiated SCC in both nude and wild-type mice (Fig. 3M). These tumors showed decreased stratification and keratinization, loss of intercellular junctions, increased nuclear/cytoplasmic ratio, nuclear pleiomorphism, and occasional mitotic figures. We examined expression of cell cycle regulatory proteins in metastatic tumors in nude and wild-type mice. PCNA was overexpressed in $50 \%$ of metastatic tumors in nude mice compared to $10 \%$ in wild-type animals
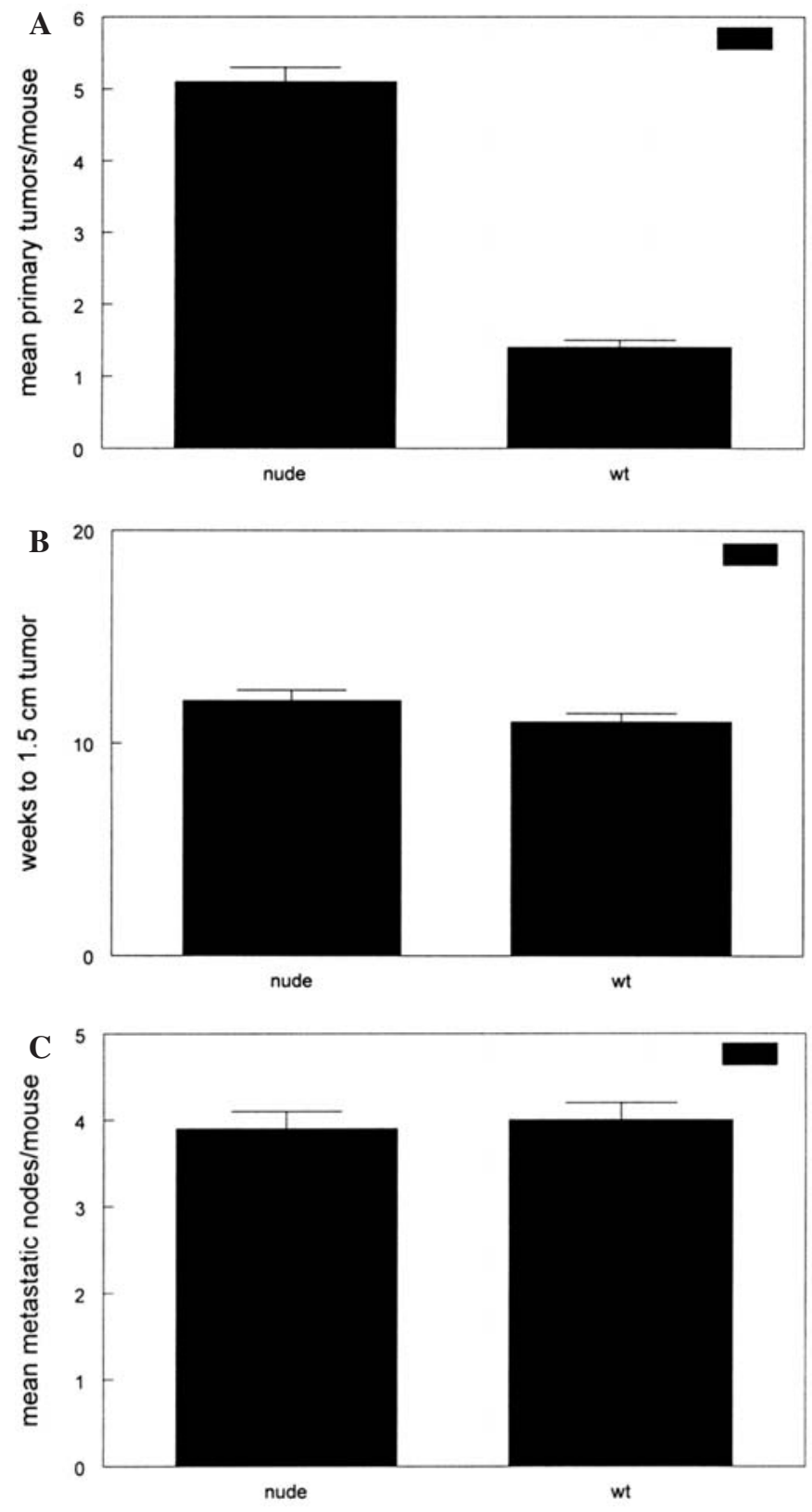

Figure 2. Increased number of tumors in a nude mouse model of head and neck cancer. Squamous cell carcinoma was induced as described in Materials and methods. (A), The total number of tumors which developed per mouse was recorded. (B), Similar growth rates in tumors arising in nude and wild-type mice. The number of weeks to achieve a $1.5-\mathrm{cm}$ tumor was recorded. (C), Similar numbers of metastatic lymph nodes in nude and wild-type mice. Cervical lymph nodes were evaluated by hematoxylin and eosin staining of serial sections. Error bars indicate SEM.

(Fig. 3N). Cyclins B and D were overexpressed in $13 \%$ of metastatic tumors in nude mice, and c-met was overexpressed in $25 \%$ of metastatic tumors in these animals which was similar to percentages observed in wild-type animals (data not shown). These results indicate that distinct differences exist between primary and metastatic tumors in the mouse model.

To characterize differences in gene expression between primary well differentiated SCC in nude and wild-type mice, we performed global gene expression profiling by microarray analysis. As shown in Table I, proteins initially characterized in salivary gland differentiation were upregulated in squamous cell carcinoma from nude mice (demilune cell and parotid 

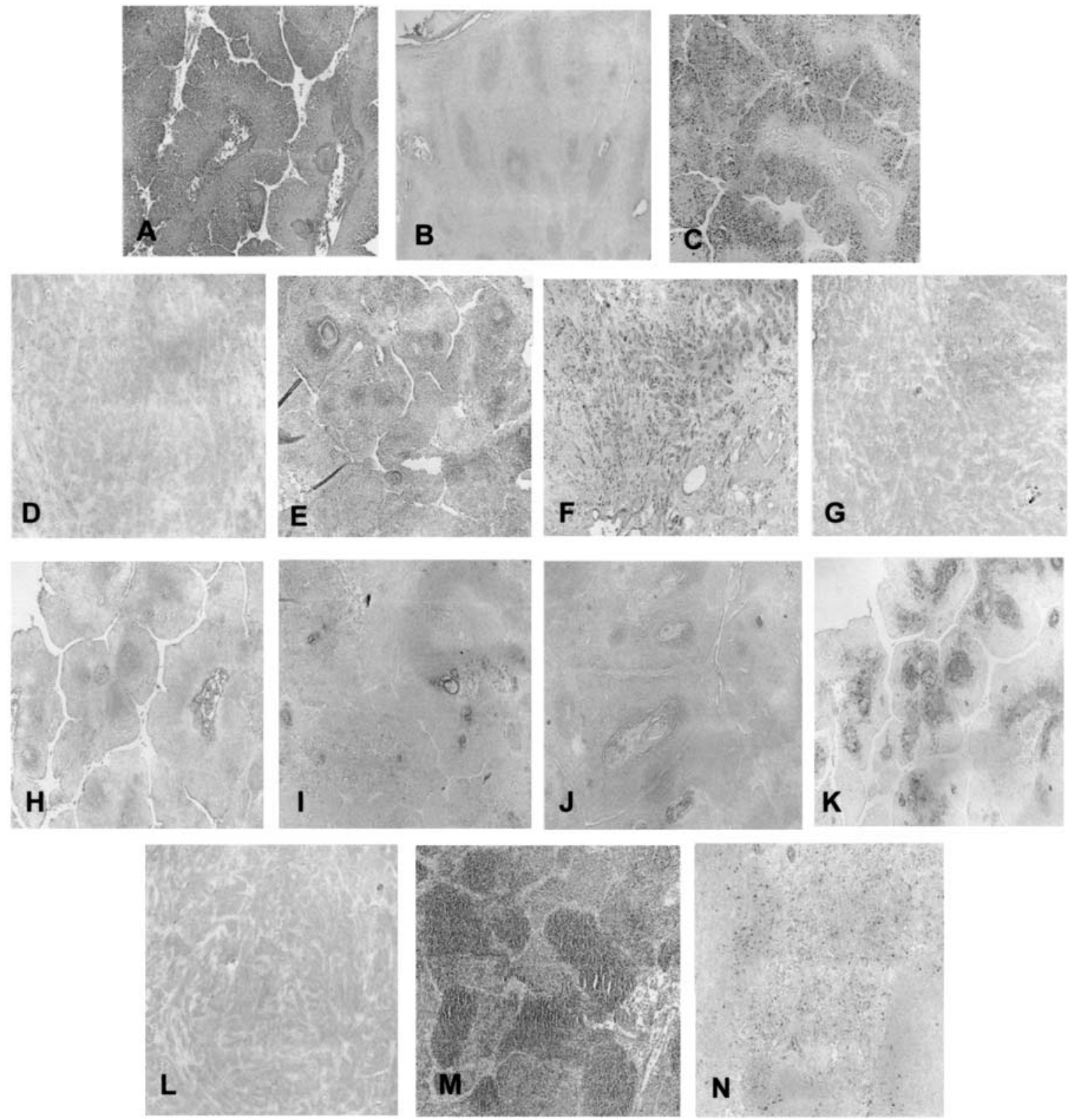

Figure 3. Expression of cell cycle regulatory proteins in squamous cell carcinoma derived from the nude mouse model. (A), Hematoxylin and eosin stained section of well differentiated SCC in the nude mouse. (B), Expression of epidermal growth factor receptor, proliferating cell nuclear antigen (C), cyclin A (D), cyclin B $(\mathrm{E})$, cyclin D (F), cyclin E (G), p16 (H), c-myc (I), hepatocyte growth factor $(\mathrm{J})$, transforming growth factor $\alpha(\mathrm{K})$, and c-met $(\mathrm{L})$ in primary SCC derived from the nude mouse model is shown by immunohistochemistry. (M), Hematoxylin and eosin stained section showing metastatic squamous carcinoma cells invading a cervical lymph node in the nude mouse model of head and neck cancer. $(\mathrm{N})$, Proliferating cell nuclear antigen expression in metastatic tumor cells in a cervical lymph node from the nude mouse model is shown by immunohistochemistry. These experiments were performed at least three times with tissue from different tumors. Representative sections are shown.

protein, 56.1-fold; cysteine-rich secretory protein 1, 43.7-fold; submaxillary gland androgen regulated protein 1, 35.1-fold). A number of the forkhead family of transcription factors were differentially regulated in SCC from nude mice (forkhead box A1, 33.8-fold; forkhead box G1, 7.6-fold; forkhead box C1, 5.7-fold; forkhead box P4, -6.3-fold). Keratins found in simple epithelia were upregulated in SCC in nude mice (keratin 19, 26.6-fold; keratin 8, 19.8-fold; keratin 18, 15.9-fold). Genes involved in terminal differentiation were downregulated in SCC from nude mice (transglutaminase 3, -26.9-fold; desmoglein 1B, -7.8-fold; corneodesmosin, -5.4-fold; keratin complex 1 , acidic, gene 5, -5.1 -fold). Inhibitory growth factor pathways were downregulated in SCC from nude mice (transforming growth factor $\beta$ receptor III, -9.3-fold; bone morphogenic protein receptor, type II, -6.8-fold; transforming growth factor $32,-5.6$-fold). The growth promoting epidermal growth factor receptor also was downregulated by 5.1-fold. Genes involved in epigenetic regulation were downregulated [Jumonji, AT rich interactive domain 2, -7.6-fold; jumonji, AT rich interactive domain 1C (Rbp2 like), -7.1-fold; p300/ CBP-associated factor, -6.9-fold; histone deacetylase 8, -6.2fold; CREB binding protein, -5.3-fold]. Genes involved in embryonic development were differentially regulated in SCC from nude mice (SRY-box containing gene 2, 18.2-fold; homeobox B3, 6.6-fold; SRY-box containing gene 9, 6.1-fold; distal-less homeobox 5,-5.3-fold). These results suggest that differentiation of HNSCC from nude mice is altered compared to those arising in wild-type mice. 
Table I. Gene expression changes between primary SCC in wild-type and nude mice (831 genes).

\begin{tabular}{|c|c|c|c|}
\hline Accession & Symbol & Gene name & Fold change \\
\hline $\mathrm{C} 86550$ & Dcpp & Demilune cell and parotid protein & 56.1 \\
\hline AV360029 & Vnn1 & Vanin 1 & 54.9 \\
\hline NM_009638 & Crisp1 & Cysteine-rich secretory protein 1 & 43.7 \\
\hline NM_011422 & Smr1 & Submaxillary gland androgen regulated protein 1 & 35.1 \\
\hline NM_008259 & Foxa1 & Forkhead box A1 & 33.8 \\
\hline AI 323288 & Id 4 & Inhibitor of DNA binding 4 & 29.2 \\
\hline NM_007812 & Cyp2a4 & Cytochrome P450, family 2 , subfamily a, polypeptide 4 & 29.1 \\
\hline NM_008471 & Krt1-19 & Keratin complex 1 , acidic, gene 19 & 26.6 \\
\hline NM_031170 & Krt2-8 & Keratin complex 2 , basic, gene 8 & 19.8 \\
\hline $\mathrm{U} 31 \overline{9} 67$ & Sox 2 & SRY-box containing gene 2 & 18.2 \\
\hline NM_010664 & Krt1-18 & Keratin complex 1 , acidic, gene 18 & 15.9 \\
\hline AW5 54594 & Prlr & Prolactin receptor & 12.1 \\
\hline AK010826 & Jam2 & Junction adhesion molecule 2 & 9.2 \\
\hline BG862223 & Camk2b & Calcium/calmodulin-dependent protein kinase II, beta & 9.0 \\
\hline BB543291 & Ccrl1 & Chemokine (C-C motif) receptor-like 1 & 9.0 \\
\hline AF333251 & $\mathrm{I} 124$ & Interleukin 24 & 8.8 \\
\hline BQ176424 & Clic6 & Chloride intracellular channel 6 & 8.7 \\
\hline AF319173 & Psca & Prostate stem cell antigen & 8.5 \\
\hline NM_011478 & Sprr3 & Small proline-rich protein 3 & 8.3 \\
\hline AW611462 & Cldn3 & Claudin 3 & 8.2 \\
\hline NM_008241 & Foxg1 & Forkhead box G1 & 7.6 \\
\hline C80220 & Rab2 & RAB2, member RAS oncogene family & 7.2 \\
\hline NM_008827 & Pgf & Placental growth factor & 7.1 \\
\hline AI 172943 & Gsta3 & Glutathione S-transferase, alpha 3 & 6.9 \\
\hline BG073383 & Hoxb3 & Homeobox B3 & 6.6 \\
\hline NM_023785 & $\mathrm{Cxcl} 7$ & Chemokine (C-X-C motif) ligand 7 & 6.4 \\
\hline $\mathrm{BC} \overline{2} 24958$ & Sox9 & SRY-box containing gene 9 & 6.1 \\
\hline NM_011146 & Pparg & Peroxisome proliferator activated receptor gamma & 5.8 \\
\hline BB759833 & Foxc1 & Forkhead box $\mathrm{C} 1$ & 5.7 \\
\hline C80147 & Hdgf & Hepatoma derived growth factor & 5.3 \\
\hline NM_008935 & Prom1 & Prominin 1 & 5.2 \\
\hline NM_011452 & Serpinb9b & Serine (or cysteine) peptidase inhibitor, clade B, $9 \mathrm{~b}$ & -5.0 \\
\hline AW538733 & Adam17 & A disintegrin and metallopeptidase domain 17 & -5.0 \\
\hline X65506 & Krt1-5 & Keratin complex 1 , acidic, gene 5 & -5.1 \\
\hline AK014017 & Egfr & Epidermal growth factor receptor & -5.1 \\
\hline BG147188 & Muc6 & Mucin 6, gastric & -5.2 \\
\hline BM217698 & Tgfbr1 & Transforming growth factor, beta receptor I & -5.2 \\
\hline NM_010056 & Dlx5 & Distal-less homeobox 5 & -5.3 \\
\hline BB475090 & Crebbp & CREB binding protein & -5.3 \\
\hline BC019788 & Plcb2 & Phospholipase C, beta 2 & -5.4 \\
\hline BM231053 & Cdsn & Corneodesmosin & -5.4 \\
\hline BB477214 & Masp1 & Mannan-binding lectin serine peptidase 1 & -5.5 \\
\hline BB296763 & Tgfb2 & Transforming growth factor, beta 2 & -5.6 \\
\hline Y17709 & Fzd9 & Frizzled homolog 9 (Drosophila) & -5.7 \\
\hline AB012278 & Cebpb & CCAAT/enhancer binding protein $(\mathrm{C} / \mathrm{EBP})$, beta & -5.8 \\
\hline BB136012 & Capg & Capping protein (actin filament), gelsolin-like & -5.8 \\
\hline AV372589 & Itga1 & Integrin alpha 1 & -6.0 \\
\hline AK004739 & Dll4 & Delta-like 4 (Drosophila) & -6.1 \\
\hline AK011332 & Hdac8 & Histone deacetylase 8 & -6.2 \\
\hline BQ286886 & Foxp4 & Forkhead box P4 & -6.3 \\
\hline NM_011691 & Vav1 & Vav1 oncogene & -6.4 \\
\hline AK020411 & Bmp7 & Bone morphogenetic protein 7 & -6.6 \\
\hline BB185152 & Bmpr2 & Bone morphogenic protein receptor, type II & -6.8 \\
\hline BM240241 & Pcaf & $\mathrm{P} 300 / \mathrm{CBP}$-associated factor & -6.9 \\
\hline BB050663 & Rora & RAR-related orphan receptor alpha & -6.9 \\
\hline BB362489 & Jarid1c & Jumonji, AT rich interactive domain $1 \mathrm{C}$ (Rbp2 like) & -7.1 \\
\hline BM293452 & Jarid2 & Jumonji, AT rich interactive domain 2 & -7.6 \\
\hline NM_008781 & Pax3 & Paired box gene 3 & -7.6 \\
\hline AY028607 & Krt2-16 & Keratin complex 2, basic, gene 16 & -7.7 \\
\hline AV253195 & Dsg1b & Desmoglein 1 beta & -7.8 \\
\hline AF039601 & Tgfbr3 & Transforming growth factor, beta receptor III & -9.3 \\
\hline BB324744 & Esrra & Estrogen related receptor, alpha & -12.1 \\
\hline BB790825 & Efna2 & Ephrin A2 & -13.9 \\
\hline AI893889 & Tgm3 & Transglutaminase 3 , E polypeptide & -26.9 \\
\hline
\end{tabular}


We also examined gene expression changes between metastatic SCC in wild-type and nude mice. As shown in Table II, 2144 differentially expressed genes were identified between metastatic SCC in wild-type and nude mice. A number of kinases were upregulated in metastatic SCC from nude mice (phosphatidylinositol-4-phosphate 5-kinase, 25.9-fold; nemo like kinase, 21.8-fold; casein kinase 1, 10.5-fold). Growth factors and receptors were upregulated in SCC from nude mice (desert hedgehog, 7.4-fold; nerve growth factor receptor, 6.9-fold; transforming growth factor $32,6.4$-fold; fibroblast growth factor 4, 6.2-fold; hepatoma derived growth factor, 5.3-fold; frizzled homolog 3, 5.2-fold). Telomere associated proteins were upregulated in SCC from nude mice (telomeric repeat binding factor 2, 9.2-fold; tankyrase, 6.0-fold). Transcription factors were also upregulated in metastatic SCC from nude mice (CCAAT/enhancer binding protein $\delta$, 9.0-fold; signal transducer and activator of transcription 3, 5.5-fold; forkhead box F1a, 5.5-fold; transformation related protein 63, 5.4-fold). Histone demethylases were differentially regulated in metastatic SCC from nude mice (Jumonji domain containing 1C, 7.4-fold; Jumonji domain containing 3, 5.8-fold; Jumonji, AT rich interactive domain $1 \mathrm{~A},-9.0$-fold; Jumonji, AT rich interactive domain $1 \mathrm{C},-10.3$-fold). Certain growth factors and receptors were downregulated in metastatic SCC from nude mice (Jagged 1, -5.2-fold; $\delta$ like 3,-5.8-fold; transforming growth factor receptor $\beta 1,-6.4$-fold; wingless related MMTV integration site 10a, -6.9-fold). Tumor suppressor expression was inhibited in metastatic SCC from nude mice (BRCA1 associated protein, -5.1-fold; retinoblastoma binding protein 6 , -7.0-fold; large tumor suppressor 2, -12.7-fold). Markers of epithelial differentiation were downregulated in metastatic SCC from nude mice (suprabasin, -6.6-fold; mucin 10, -7.4-fold; desmoplakin, -8.1 -fold). Cell cycle regulatory genes were inhibited in metastatic SCC from nude mice (cyclin D2, -7.5-fold; cyclin dependent kinase inhibitor 1B, -14.0-fold). Specific transcription factors also were downregulated in metastatic SCC from nude mice (retinoid X receptor $\gamma,-5.2$-fold; forkhead box Q1, -5.6-fold; Jun proto-oncogene related gene D1, -6.1-fold; Vav3 oncogene, -10.4-fold; lymphoid enhancer factor 1, -12.7-fold; myeloblastosis oncogene, -57.8-fold). These gene expression changes suggest that metastatic SCC from nude mice may be more biologically aggressive than their counterparts in wild-type mice.

In previously published study, we examined differential gene expression between primary and metastatic tumors in wild-type mice (23). In the present study, we examined gene expression differences between primary and metastatic tumors in nude mice. As shown in Table III, chemokine ligands and receptors were upregulated in metastatic SCC compared to primary tumors (Cxc19, 37.5-fold; Ccl19, 27.2-fold; Cxcl13, 17.0-fold; Ccl8, 13.5-fold; Ccl5, 11.2-fold; Cxcl10, 7.6-fold; Ccr6, 5.7-fold; Cxcl12, 5.0-fold). Signal transduction genes also were upregulated in metastatic SCC (Ras related C3 botulinum substrate 2, 20.3-fold; signal transducer and activator of transcription 1, 12.6-fold; growth factor receptor bound protein 2, 6.3-fold; c-src tyrosine kinase, 5.8-fold). Growth factors and receptors were downregulated in metastatic SCC compared to primary tumors in nude mice (heparin binding EGF like growth factor, -5.0-fold; epidermal growth factor, -5.1-fold; smoothened homolog, -5.3-fold; v-erb-b2 erythro- blastic leukemia viral oncogene, -6.5-fold; bone morphogenetic protein 1, -7.0-fold; amphiregulin, -7.8-fold; growth hormone receptor, -8.9-fold; Jagged 1, -10.1-fold; connective tissue growth factor, -24.8-fold). Epithelial differentiation markers were downregulated in metastatic SCC (vitamin D receptor, -6.7-fold; loricrin, -13.1-fold; keratin 8, -15.8-fold; desmoglein 3, -20.0-fold; keratin 18, -21.7-fold; keratin 16, -23.0-fold; suprabasin, -39.1-fold; desmoplakin, -45.0-fold; keratin 10, -50.5-fold; keratin 6a, -90.5-fold; keratin 5, -94.6-fold; small proline rich protein 2A, -110.9-fold; keratin 14, -123.1-fold). Peptidase inhibitor gene expression was down-regulated in metastatic SCC (serpin E1, -5.4-fold; serine peptidase inhibitor 5, -6.7-fold; tissue inhibitor of metalloproteinase 1, -12.0 -fold). These gene expression changes are consistent with metastatic phenotype in the nude mouse model of SCC.

We wondered how lymphocytes in cervical lymph nodes responded to metastatic tumor cells. We performed differential gene expression on wild-type lymphocytes from non-tumor bearing mice and those in contact with metastatic tumor cells in lymph nodes. As shown in Table IV, the major class of genes upregulated in lymphocytes in contact with metastatic tumor cells was immunoglobin chains [immunoglobulin $\kappa$ light chain 17-1A, 80.5-fold; immunoglobulin $\kappa$ chain variable 21, 70.3-fold; Ig rearranged heavy chain mRNA VH-DH-JH1 region, 50.4-fold; Ig $\kappa$ chain, 47.3-fold; immunoglobulin heavy chain $\gamma$ polypeptide, 42.8-fold; immunoglobulin heavy chain J558 family, 33.1-fold; immunoglobulin $\kappa$ chain variable 32, 29.3-fold; similar to immunoglobulin light chain, 23.1-fold; immunoglobulin $\kappa$ chain constant region, 18.6-fold; immunoglobulin $\kappa$ chain variable 28,15 .8-fold; immunoglobulin heavy chain 4 (serum $\operatorname{IgG1}$ ), 11.3-fold; immunoglobulin $\lambda$ chain, variable 1, 5.8-fold; immunoglobulin heavy chain 1a (serum IgG2a), 5.5-fold]. A number of other lymphocyte genes were upregulated in tumor cell exposed lymphocytes (lymphocyte antigen 9, 17.8-fold; pre-B lymphocyte gene 1, 11.3-fold; lymphocyte enhancer binding factor 1, 9.6-fold). Toll like receptor signaling was upregulated in tumor cell exposed lymphocytes (toll like receptor 4, 9.8-fold; toll like receptor 12, 6.3-fold; toll like receptor adaptor molecule 1, 6.2-fold; toll interleukin 1 receptor domain adaptor protein, 5.2-fold). Cell death genes were upregulated in tumor cell exposed lymphocytes (BH3 interacting domain death agonist, 11.2-fold; programmed cell death 1, 10.3-fold; caspase 7, 7.0-fold; caspase recruitment domain 4, 5.7-fold). A number of lymphocyte survival factors were downregulated in tumor cell exposed lymphocytes (BCL2 like 12, -20.2-fold; myeloproliferative leukemia virus oncogene, -19.1-fold; plasmacytoma expressed transcript 2, -14.2-fold; B-cell CLL/lymphoma 7A, -14.1fold; B cell leukemia/lymphoma 11B, -11.3-fold; early B cell factor 3, -8.3-fold; pre B cell leukemia transcription factor 4, -8.2-fold; B cell translocation gene 4, -7.1-fold; pre B cell leukemia transcription factor 1, -6.1-fold; T cell lymphoma invasion and metastasis 2, -5.9-fold; inhibitor of Bruton agammaglobulinemia tyrosine kinase, -5.8-fold; B cell CLL/ lymphoma 9 like, -5.4-fold; B cell leukemia/lymphoma 2 related protein $\mathrm{A} 1 \mathrm{a},-5.1$-fold). These results indicate that lymphocytes react to metastatic tumor cells by upregulating immunoglobin gene expression but are prone to apoptosis via decreased expression of survival factors and upregulation of cell death genes. 
Table II. Gene expression changes between metastatic SCC in wild-type and nude mice (2144 genes).

\begin{tabular}{|c|c|c|c|}
\hline Accession & Symbol & Gene name & Fold change \\
\hline AW495875 & Ptp4a2 & Protein tyrosine phosphatase $4 \mathrm{a} 2$ & 26.6 \\
\hline AI447325 & Pip5k2b & Phosphatidylinositol-4-phosphate 5-kinase, type II, beta & 25.9 \\
\hline AU022700 & Arnt & Aryl hydrocarbon receptor nuclear translocator & 23.3 \\
\hline BB636266 & Nlk & Nemo like kinase & 21.8 \\
\hline NM_021518 & Rab2 & RAB2, member RAS oncogene family & 17.0 \\
\hline BE446893 & Igfbp7 & Insulin-like growth factor binding protein 7 & 15.1 \\
\hline BB543291 & Ccrl1 & Chemokine (C-C motif) receptor-like 1 & 13.5 \\
\hline AI451488 & Csnk1g1 & Casein kinase 1 , gamma 1 & 10.5 \\
\hline AK017392 & Ppp1cb & Protein phosphatase 1 , catalytic subunit, beta isoform & 9.4 \\
\hline BE954012 & Terf2 & Telomeric repeat binding factor 2 & 9.2 \\
\hline AI642132 & Cebpd & CCAAT/enhancer binding protein $(\mathrm{C} / \mathrm{EBP})$, delta & 9.0 \\
\hline AV257260 & $\mathrm{Plcd} 4$ & Phospholipase C, delta 4 & 7.5 \\
\hline AV367068 & Dhh & Desert hedgehog & 7.4 \\
\hline BB667902 & Jmjd1c & Jumonji domain containing $1 \mathrm{C}$ & 7.4 \\
\hline BB790825 & Efna2 & Ephrin A2 & 6.9 \\
\hline BI151406 & Ngfr & Nerve growth factor receptor & 6.9 \\
\hline BB296763 & Tgfn2 & Transforming growth factor, beta 2 & 6.4 \\
\hline NM_007939 & Epha8 & Eph receptor A8 & 6.3 \\
\hline ВМ'̄93059 & Pten & Phosphatase and tensin homolog & 6.2 \\
\hline BB709552 & Fgf4 & Fibroblast growth factor 4 & 6.2 \\
\hline BB645478 & Tnks & Tankyrase & 6.0 \\
\hline BG228765 & Jmjd3 & Jumonji domain containing 3 & 5.8 \\
\hline BC003806 & Stat3 & Signal transducer and activator of transcription 3 & 5.5 \\
\hline NM_010426 & Foxf1a & Forkhead box F1a & 5.5 \\
\hline X99143 & Krt2-10 & Keratin complex 2, basic, gene 10 & 5.4 \\
\hline AF075436 & Trp63 & Transformation related protein 63 & 5.4 \\
\hline C80147 & Hdgf & Hepatoma-derived growth factor & 5.3 \\
\hline AU020229 & Fzd3 & Frizzled homolog 3 (Drosophila) & 5.2 \\
\hline BM238599 & $\mathrm{Vcl}$ & Vinculin & 5.2 \\
\hline BF467164 & Mta1 & Metastasis associated 1 & 5.1 \\
\hline NM_028227 & Brap & BRCA1 associated protein & -5.1 \\
\hline NM_009107 & Rxrg & Retinoid X receptor gamma & -5.2 \\
\hline NM_008239 & Foxq1 & Forkhead box Q1 & -5.6 \\
\hline $\mathrm{AB} 013440$ & $\mathrm{D} 113$ & Delta-like 3 (Drosophila) & -5.8 \\
\hline AJ318863 & $\mathrm{Ccrl} 2$ & Chemokine (C-C motif) receptor-like 2 & -5.9 \\
\hline BM122502 & Jund1 & Jun proto-oncogene related gene d1 & -6.1 \\
\hline AA880220 & Jag1 & Jagged 1 & -6.2 \\
\hline BM217698 & Tgfbr1 & Transforming growth factor, beta receptor I & -6.4 \\
\hline M64429 & Braf & Braf transforming gene & -6.5 \\
\hline AI507307 & Sbsn & Suprabasin & -6.6 \\
\hline BB475090 & Crebbp & CREB binding protein & -6.8 \\
\hline NM_009518 & Wnt10a & Wingless related MMTV integration site 10a & -6.9 \\
\hline AI428101 & Sox4 & SRY-box containing gene 4 & -7.0 \\
\hline BB367420 & Rbbp6 & Retinoblastoma binding protein 6 & -7.0 \\
\hline BB284583 & Itga4 & Integrin alpha 4 & -7.1 \\
\hline NM_008644 & Muc10 & Mucin 10 , submandibular gland salivary mucin & -7.4 \\
\hline NM_009829 & Ccnd2 & Cyclin D2 & -7.5 \\
\hline BM9 47855 & Plk3 & Polo-like kinase 3 (Drosophila) & -7.5 \\
\hline AV297961 & Dsp & Desmoplakin & -8.1 \\
\hline AK002609 & Sirt5 & Sirtuin 5 & -8.8 \\
\hline AK011603 & Bcas & Breast carcinoma amplified sequence 3 & -8.8 \\
\hline BB376407 & Jarid1a & Jumonji, AT rich interactive domain 1A (Rbp2 like) & -9.0 \\
\hline NM_033573 & Prcc & Papillary renal cell carcinoma (translocation-associated) & -9.2 \\
\hline AF146523 & Malat1 & Metastasis associated lung adenocarcinoma transcript 1 & -9.9 \\
\hline BB362489 & Jarid1c & Jumonji, AT rich interactive domain 1C (Rbp2 like) & -10.3 \\
\hline $\mathrm{BC} 027242$ & Vav3 & Vav3 oncogene & -10.4 \\
\hline $\mathrm{BC} 003261$ & Aurkb & Aurora kinase B & -12.3 \\
\hline BE986745 & Lats2 & Large tumor suppressor 2 & -12.7 \\
\hline NM_010703 & Lef1 & Lymphoid enhancer factor 1 & -12.7 \\
\hline BF783030 & Fzd4 & Frizzled homolog 4 (Drosophila) & -12.7 \\
\hline NM_009875 & Cdkn1b & Cyclin-dependent kinase inhibitor 1B (p27) & -14.0 \\
\hline M80360 & Msh3 & MutS homolog 3 (E. coli) & -24.7 \\
\hline NM_033597 & Myb & Myeloblastosis oncogene & -57.8 \\
\hline
\end{tabular}


Table III. Gene expression changes between primary and metastatic SCC in nude mice (1205 genes).

\begin{tabular}{|c|c|c|c|}
\hline Accession & Symbol & Gene name & Fold change \\
\hline BC014718 & Dnase & Deoxyribonuclease I & 70.4 \\
\hline NM_008599 & Cxc19 & Chemokine (C-X-C motif) ligand 9 & 37.5 \\
\hline NM_011888 & Ccl19 & Chemokine (C-C motif) ligand 19 & 27.2 \\
\hline NM_009008 & Rac2 & RAS-related C3 botulinum substrate 2 & 20.3 \\
\hline AF030636 & Cxcl13 & Chemokine (C-X-C motif) ligand 13 & 17.0 \\
\hline NM_021443 & $\mathrm{Ccl} 8$ & Chemokine (C-C motif) ligand 8 & 13.5 \\
\hline AW2 14029 & Stat1 & Signal transducer and activator of transcription 1 & 12.6 \\
\hline NM_013653 & $\mathrm{Ccl} 5$ & Chemokine (C-C motif) ligand 5 & 11.2 \\
\hline NM_008404 & Itgb2 & Integrin beta 2 & 8.5 \\
\hline BE685667 & Ccnd3 & Cyclin D3 & 8.1 \\
\hline NM_009515 & Was & Wiskott-Aldrich syndrome homolog (human) & 7.6 \\
\hline NM_021274 & Cxcl10 & Chemokine (C-X-C motif) ligand 10 & 7.6 \\
\hline BG064712 & Grb2 & Growth factor receptor bound protein 2 & 6.3 \\
\hline BG094076 & Csk & $\mathrm{C}$-src tyrosine kinase & 5.8 \\
\hline NM_009835 & Ccr6 & Chemokine (C-C motif) receptor 6 & 5.7 \\
\hline $\mathrm{BC} 016492$ & Tgm2 & Transglutaminase $2, \mathrm{C}$ polypeptide & 5.5 \\
\hline BM220820 & Foxp1 & Forkhead box P1 & 5.4 \\
\hline BC006640 & $\mathrm{Cxcl} 12$ & Chemokine (C-X-C motif) ligand 12 & 5.0 \\
\hline L07264 & Hbegf & Heparin-binding EGF-like growth factor & -5.0 \\
\hline AV369812 & Egfr & Epidermal growth factor receptor & -5.1 \\
\hline BB543979 & Itgb5 & Integrin beta 5 & -5.1 \\
\hline BB364488 & Foxo3a & Forkhead box O3a & -5.3 \\
\hline AW555326 & Smo & Smoothened homolog (Drosophila) & -5.3 \\
\hline NM_008871 & Serpine1 & Serine (or cysteine) peptidase inhibitor, clade E, 1 & -5.4 \\
\hline AV074236 & S100a16 & S100 calcium binding protein A16 & -5.4 \\
\hline AF059567 & Cdkn2b & Cyclin-dependent kinase inhibitor 2B (p15) & -5.6 \\
\hline NM_007631 & Ccnd1 & Cyclin D1 & -5.7 \\
\hline NM_013599 & Mmp9 & Matrix metallopeptidase 9 & -5.8 \\
\hline $\mathrm{BC} 027242$ & Vav3 & Vav 3 oncogene & -5.9 \\
\hline BC004663 & Dsc2 & Desmocollin 2 & -5.9 \\
\hline BF147716 & Mmp2 & Matrix metallopeptidase 2 & -6.2 \\
\hline BB759833 & Foxc1 & Forkhead box $\mathrm{C} 1$ & -6.3 \\
\hline NM_009821 & Runx1 & Runt related transcription factor 1 & -6.4 \\
\hline X66083 & $\mathrm{Cd} 44$ & CD44 antigen & -6.5 \\
\hline BF140685 & Erbb3 & V-erb-b2 erythroblastic leukemia viral oncogene & -6.5 \\
\hline AV241297 & Spink5 & Serine peptidase inhibitor, Kazal type 5 & -6.7 \\
\hline AV290079 & $\mathrm{Vdr}$ & Vitamin D receptor & -6.7 \\
\hline NM_007393 & Actb & Actin, beta, cytoplasmic & -6.9 \\
\hline BI465857 & Klf5 & Kruppel-like factor 5 & -6.9 \\
\hline $\mathrm{L} 24755$ & Bmp1 & Bone morphogenetic protein 1 & -7.0 \\
\hline NM_013496 & Crabp1 & Cellular retinoic acid binding protein I & -7.1 \\
\hline BQ173967 & Efna5 & Ephrin A5 & -7.5 \\
\hline NM_009704 & Areg & Amphiregulin & -7.8 \\
\hline NM_010284 & Ghr & Growth hormone receptor & -8.9 \\
\hline NM_007913 & Egr1 & Early growth response 1 & -9.1 \\
\hline AV359819 & Jag1 & Jagged 1 & -10.1 \\
\hline BG970109 & Lamb1-1 & Laminin B1 subunit 1 & -10.2 \\
\hline ВC024958 & Sox 9 & SRY-box containing gene 9 & -10.3 \\
\hline AV026617 & Fos & FBJ osteosarcoma oncogene & -11.8 \\
\hline BC008107 & Timp1 & Tissue inhibitor of metalloproteinase 1 & -12.0 \\
\hline NM_008508 & Lor & Loricrin & -13.1 \\
\hline BM935811 & Itga6 & Integrin alpha 6 & -14.3 \\
\hline M21836 & Krt2-8 & Keratin complex 2 , basic, gene 8 & -15.8 \\
\hline BB040443 & Snai2 & Snail homolog 2 (Drosophila) & -19.6 \\
\hline AV229522 & Dsg3 & Desmoglein 3 & -20.0 \\
\hline NM_010664 & Krt1-18 & Keratin complex 1 , acidic, gene 18 & -21.7 \\
\hline NM_008470 & Krt1-16 & Keratin complex 1 , acidic, gene 16 & -23.0 \\
\hline NM_010217 & Ctgf & Connective tissue growth factor & -24.8 \\
\hline AI $8 \overline{4} 4734$ & Sbsn & Suprabasin & -39.1 \\
\hline AV297961 & Dsp & Desmoplakin & -45.0 \\
\hline AK014360 & Krt1-10 & Keratin complex 1 , acidic, gene 10 & -50.5 \\
\hline NM_008476 & Krt2-6a & Keratin complex 2 , basic, gene $6 a$ & -90.5 \\
\hline $\mathrm{BC} 006780$ & Krt2-5 & Keratin complex 2, basic, gene 5 & -94.6 \\
\hline AV371678 & Sprr2a & Small proline-rich protein $2 \mathrm{~A}$ & -110.9 \\
\hline $\mathrm{BC} 011074$ & Krt1-14 & Keratin complex 1 , acidic, gene 14 & -123.1 \\
\hline
\end{tabular}


Table IV. Gene expression changes between normal node and metastatic SCC node lymphocytes (840 genes).

\begin{tabular}{|c|c|c|c|}
\hline Accession & Symbol & Gene name & Fold change \\
\hline L41881 & LOC213481 & Immunoglobulin kappa light chain $17-1 \mathrm{~A}$ & 80.5 \\
\hline Z95478 & Igk-V1 & Immunoglobulin kappa chain variable 21 (V21) & 70.3 \\
\hline Z95476 & - & Ig rearranged heavy chain mRNA VH-DH-JH1 region & 50.4 \\
\hline U29768 & $\operatorname{IgM}$ & Ig kappa chain & 47.3 \\
\hline S69212 & Ighg & Immunoglobulin heavy chain (gamma polypeptide) & 42.8 \\
\hline AF065324 & Igh-VJ558 & Immunoglobulin heavy chain (J558 family) & 33.1 \\
\hline NM_007646 & $\mathrm{Cd} 38$ & CD38 antigen & 32.8 \\
\hline $\mathrm{U} 25103$ & Igk-V32 & Immunoglobulin kappa chain variable 32 (V32) & 29.3 \\
\hline AF099052 & $\mathrm{Ccl} 20$ & Chemokine (C-C motif) ligand 20 & 23.7 \\
\hline U55641 & - & Similar to immunoglobulin light chain & 23.1 \\
\hline BF301241 & Igk-C & Immunoglobulin kappa chain, constant region & 18.6 \\
\hline NM_008534 & Ly9 & Lymphocyte antigen 9 & 17.8 \\
\hline AB007986 & Igk-V28 & Immunoglobulin kappa chain variable 28 (V28) & 15.8 \\
\hline NM 010824 & Mpo & Myeloperoxidase & 13.8 \\
\hline NM_008920 & Prg2 & Proteoglycan 2 , bone marrow & 11.8 \\
\hline NM_016982 & Vpreb1 & Pre-B lymphocyte gene 1 & 11.3 \\
\hline AF466769 & Igh-4 & Immunoglobulin heavy chain 4 (serum IgG1) & 11.3 \\
\hline NM_007544 & Bid & $\mathrm{BH} 3$ interacting domain death agonist & 11.2 \\
\hline NM_008798 & Pdcd & Programmed cell death 1 & 10.3 \\
\hline AF185285 & Tlr4 & Toll-like receptor 4 & 9.8 \\
\hline NM_010703 & Lef1 & Lymphoid enhancer binding factor 1 & 9.6 \\
\hline NM_008840 & Pik3cd & Phosphatidylinositol 3-kinase catalytic delta polypeptide & 9.5 \\
\hline AF232024 & Ly6i & Lymphocyte antigen 6 complex, locus I & 9.4 \\
\hline NM_009892 & Chi313 & Chitinase 3-like 3 & 9.0 \\
\hline NM_007782 & Csf3r & Colony stimulating factor 3 receptor (granulocyte) & 8.2 \\
\hline NM 010780 & Mcpt5 & Mast cell protease 5 & 7.3 \\
\hline U67321 & Casp7 & Caspase 7 & 7.0 \\
\hline NM_011093 & Pira1 & Paired-Ig-like receptor A1 & 6.4 \\
\hline NM_010376 & $\mathrm{H} 13$ & Histocompatibility 13 & 6.4 \\
\hline NM_007572 & C1qa & Complement component 1 , q subcomponent, alpha & 6.3 \\
\hline BB745017 & Tlr12 & Toll-like receptor 12 & 6.3 \\
\hline BB452539 & Ticam 1 & Toll-like receptor adaptor molecule 1 & 6.2 \\
\hline NM_023517 & Tnfsf13 & Tumor necrosis factor (ligand) superfamily, member 13 & 6.2 \\
\hline BG072012 & Thpok & $\mathrm{T}$ helper-inducing POZ/Krueppel factor & 6.2 \\
\hline BB525754 & Ikbkb & Inhibitor of kappaB kinase beta & 5.9 \\
\hline BI676554 & Igl-VI & Immunoglobulin lambda chain, variable 1 & 5.8 \\
\hline BB138330 & Card4 & Caspase recruitment domain 4 & 5.7 \\
\hline $\mathrm{BC} 018365$ & Igh-1a & Immunoglobulin heavy chain 1 a (serum IgG2a) & 5.5 \\
\hline $\mathrm{BC} 010602$ & H2-Q1 & Histocompatibility $2, \mathrm{Q}$ region locus 1 & 5.4 \\
\hline BB800282 & Pfc & Properdin factor, complement & 5.2 \\
\hline NM_054096 & Tirap & Toll-interleukin 1 receptor domain adaptor protein & 5.2 \\
\hline NM_008694 & Ngp & Neutrophilic granule protein & 5.1 \\
\hline AF240358 & Clec4n & C-type lectin domain family 4 , member $\mathrm{n}$ & 5.1 \\
\hline BB667813 & Mlf1ip & Myeloid leukemia factor 1 interacting protein & -5.0 \\
\hline AV245981 & Igsf1 & Immunoglobulin superfamily, member 1 & -5.0 \\
\hline BI466363 & Brca1 & Breast cancer 1 & -5.0 \\
\hline NM_009337 & Tcl1 & T-cell lymphoma breakpoint 1 & -5.0 \\
\hline AF342896 & Klrb1d & Killer cell lectin-like receptor subfamily B member 1D & -5.1 \\
\hline NM_010416 & Hemt 1 & Hematopoietic cell transcript 1 & -5.1 \\
\hline AF289078 & Nfatc2 & Nuclear factor of activated T-cells, cytoplasmic, 2 & -5.1 \\
\hline AI326167 & $\mathrm{Bcl} 2 \mathrm{a} 1 \mathrm{a}$ & B-cell leukemia/lymphoma 2 related protein A1a & -5.1 \\
\hline AA119055 & Tcrg-V4 & T-cell receptor gamma, variable 6 & -5.2 \\
\hline AV294178 & Klrb1d & Killer cell lectin-like receptor subfamily B member 1D & -5.2 \\
\hline X60958 & $\mathrm{Cd} 80$ & CD80 antigen & -5.2 \\
\hline AI481991 & H2-BI & Histocompatibility 2 & -5.3 \\
\hline BF464707 & $\mathrm{Bcl} 91$ & B-cell CLL/lymphoma 9-like & -5.4 \\
\hline C81413 & Igbp1 & Immunoglobulin (CD79A) binding protein 1 & -5.6 \\
\hline $\mathrm{X} 03052$ & $\mathrm{H} 2-\mathrm{T} 18$ & Histocompatibility $2, \mathrm{~T}$ region locus 18 & -5.7 \\
\hline BE981385 & Ibtk & Inhibitor of Bruton agammaglobulinemia tyrosine kinase & -5.8 \\
\hline AY059394 & $\operatorname{Igsf} 4 \mathrm{c}$ & Immunoglobulin superfamily, member $4 \mathrm{C}$ & -5.9 \\
\hline AI552177 & Tiam2 & T-cell lymphoma invasion and metastasis 2 & -5.9 \\
\hline X03019 & Csf2 & Colony stimulating factor 2 (granulocyte-macrophage) & -6.0 \\
\hline NM 008783 & $\mathrm{Pbx} 1$ & Pre B-cell leukemia transcription factor 1 & -6.1 \\
\hline AI 847494 & Leng9 & Leukocyte receptor cluster (LRC) member 9 & -6.1 \\
\hline
\end{tabular}


Table IV. Continued.

\begin{tabular}{llll}
\hline Accession & Symbol & \multicolumn{1}{c}{ Gene name } & Fold change \\
\hline M16810 & H2-DI & Histocompatibility 2, D region locus 1 & -6.4 \\
AW259474 & Ighmbp2 & Immunoglobulin mu binding protein 2 & -6.6 \\
BB333386 & Igsf4d & Immunoglobulin superfamily, member 4 & -6.7 \\
NM_008463 & Klra5 & Killer cell lectin-like receptor, subfamily A, member 5 & -6.9 \\
NM_010702 & Lect2 & Leukocyte cell-derived chemotaxin 2 & -7.0 \\
BM225534 & Btg4 & B-cell translocation gene 4 & -7.1 \\
BB155514 & - & H-12.C4 T cell receptor alpha chain (V alpha 13 family) \\
BM941868 & Csf2ra & Colony stimulating factor 2 receptor, alpha & -7.3 \\
NM_030555 & Pbx4 & Pre-B-cell leukemia transcription factor 4 & -7.9 \\
AV244034 & Ebf3 & Early B-cell factor 3 & -8.2 \\
BM877183 & Bcl11b & B-cell leukemia/lymphoma 11B & -8.3 \\
BC017640 & Bcl7a & B-cell CLL/lymphoma 7A & -11.3 \\
NM_008821 & Pet2 & Plasmacytoma expressed transcript 2 & -14.1 \\
NM_010823 & Mpl & Myeloproliferative leukemia virus oncogene & -14.2 \\
AK013411 & Bcl2112 & BCL2-like 12 (proline rich) & -19.1 \\
\hline
\end{tabular}

\section{Discussion}

Our results indicate that decreased $\mathrm{T}$ lymphocyte function results in decreased tumor latency and increased numbers of tumors. These results suggest that $\mathrm{T}$ lymphocytes are key to elimination of tumorigenic clones. There is a large body of evidence that cytotoxic $\mathrm{T}$ lymphocytes are involved in the clinical course of cancer (reviewed in ref. 25). A variety of stimuli have been proposed to activate the immune response in cancer, including genotoxic stress induced by carcinogen exposure (reviewed in ref. 26). In early stage tongue cancer, tumors were infiltrated by cytotoxic $\mathrm{T}$ lymphocytes and natural killer cells (27). No intraepithelial tumor cells expressed the proliferation marker Ki-67 but did express the inhibitory receptor PD-1. These results indicate that lymphocytes which interact with tumor cells become suppressed or inactivated.

It was recently demonstrated in a mouse model that tumors do not escape recognition but induce immune tolerance (28). Vaccinated mice remained tumor-free but naïve mice developed a progressively growing tumor. Despite specific recognition by $\mathrm{T}$ lymphocytes, tumors did not lose immunogenicity and were rejected when transplanted to immunocompetent recipients. Tumor induced tolerance was associated with expansion of non-functional $\mathrm{T}$ lymphocytes. A variety of tumor derived factors contribute to immunosuppressive networks including VEGF, interleukin 10, TGFß, prostaglandin E, and Fas ligand (reviewed in ref. 29). These factors may be active at distant sites, thereby promoting invasion and metastasis. VEGF recruits bone marrow cells to become immature dendritic cells and macrophages. These immature cells may suppress roving dendritic cells and T lymphocytes. Soluble Fas ligand may help tumor cells escape lysis by cytotoxic $\mathrm{T}$ lymphocytes and natural killer cells by inducing apoptosis in the immune cells. In a mouse chemical carcinogenesis model, it was demonstrated that equilibrium was mechanistically distinguishable from elimination and escape (30). Neoplastic cells in this model were transformed but proliferated poorly in vivo. These cells were unedited in equilibrium but are edited when they escape immune control and become tumors. These results highlight the complex interplay between cancer cells and the immune system.
We determined that the largest class of upregulated genes in lymphocytes exposed to metastatic HNSCC was immunoglobulin genes. The antigens arising during the course of the disease may derive from somatic mutations in normal gene products, tumor specific antigens shared among patients with a particular type of cancer, normal tissue specific gene products, and normal proteins predominantly expressed by tumors (31-34). However, lymphocytes exposed to metastatic tumor cells were prone to apoptosis via decreased expression of survival factors and upregulation of cell death genes. These results suggest that lymphocytes are primed for elimination by interaction with metastatic tumor cells.

Primary and metastatic tumors arising in nude mice were significantly different from those in wild-type animals. Differentiation of HNSCC from nude mice is shifted towards secretory genes compared to those arising in wild-type mice. Comparison of gene expression in metastatic tumors in these two models indicated that metastatic SCC from nude mice may be more biologically aggressive than their counterparts in wild-type mice. However, these differences were not apparent in the number of lymph nodes containing metastatic tumor cells. Expression of cell cycle regulatory proteins examined by immunohistochemistry revealed important similarities with human HNSCC characterized in our previously published study (24). Future studies will determine how these signaling pathways regulate metastatic phenotype and immune escape in HNSCC.

\section{References}

1. Moore SR, Johnson NW, Pierce AM, et al: The epidemiology of mouth cancer: a review of global incidence. Oral Dis 6: 65-74, 2000.

2. Goldberg HI, Lockwood SA, Wyatt SW, et al: Trends and differentials in mortality from cancers of the oral cavity and pharynx in the United States. Cancer 74: 565-572, 1994.

3. Hernandez G, Arriba L, Jimenez C, et al: Rapid progression from oral leukoplakia to carcinoma in an immunosuppressed liver transplant recipient. Oral Oncol 39: 87-90, 2003.

4. King GN, Healy CM, Glover MT, et al: Increased prevalence of dysplastic and malignant lip lesions in renal transplant patients. N Eng J Med 332: 1052-1057, 1995.

5. De Visscher JG, Bouwes Bavinck JN and van der Waal I: Squamous cell carcinoma of the lower lip in renal transplant recipients. Int J Oral Maxillofac Surg 26: 120-123, 1997. 
6. Bilinska-Pietraszek E, Namyslowski G, Mrowka-Kata K, Scierski W and Aniol-Borkowska M: A case of tongue neoplasm in a 15 year old patient treated with immunosuppressants for renal insufficiency. Otolaryngol Pol 55: 95-97, 2001.

7. Van Zuuren EJ, De Visscher JG and Bouwes Bavinck JN: Carcinoma of the lip in kidney transplant recipients. J Am Acad Dermatol 38: 497-499, 1998.

8. Bhatia S, Louie AD, Bhatia R, et al: Solid cancers after bone marrow transplantation. J Clin Oncol 19: 464-471, 2001.

9. Jewett A, Head C and Cacalano NA: Emerging mechanisms of immunosuppression in oral cancers. J Dent Res 85: 1061-1073, 2006.

10. Dunn GP, Bruce AT, Ikeda H, Old LJ and Schreiber RD: Cancer immunoediting: from immunosurveillance to tumor escape. Nat Immunol 3: 991-998, 2002.

11. Dunn GP, Old LJ and Schreiber RD: The immunobiology of cancer immunosurveillance and immunoediting. Immunity 21 : 137-148, 2004.

12. Laad A, Kode J, Chavan S, Rao R, Fakih AR and Chiplunkar S: Limiting dilution analysis of proliferating and cytotoxic lymphocytes in the peripheral blood and tumors of oral cancer patients. Eur J Cancer 32: 337-342, 1996.

13. Jewett A, Cacalano NA, Head C and Teruel A: Coengagement of CD16 and CD94 receptors mediates secretion of chemokines and induces apoptotic death of native natural killer cells. Clin Cancer Res 12: 1994-2003, 2006.

14. Thomas DW, Matthews JB, Patel V, Game SM and Prime SS: Inflammatory cell infiltrate associated with primary and transplanted tumors in an inbred model of oral carcinogenesis. J Oral Pathol Med 24: 23-31, 1995.

15. Schantz SP, Shillitoe EJ, Brown B and Campbell B: Natural killer cell activity and head and neck cancer: a clinical assessment. J Natl Cancer Inst 77: 869-875, 1986.

16. Hoffmann TK, Donnenberg AD, Finkelstein SD, et al: Frequencies of tetramer+ $\mathrm{T}$ cells specific for the wild type sequence p53(264-272) peptide in the circulation of patients with head and neck cancer. Cancer Res 62: 3521-3529, 2002.

17. Whiteside TL, Letessier E, Hirabayashi H, et al: Evidence for local and systemic activation of immune cells by peritumoral injections of interleukin 2 in patients with advanced squamous cell carcinoma of the head and neck. Cancer Res 53: 5654-5662, 1993.

18. Whiteside TL, Chikamatsu K, Nagashima S and Okada K: Antitumor effects of cytolytic T lymphocytes (CTL) and natura killer (NK) cells in head and neck cancer. Anticancer Res 16: 2357-2364, 1996.

19. Han X, Papadopoulos AJ, Ruparelia V, Devaja O and Raju KS: Tumor lymphocytes in patients with advanced ovarian cancer: changes during in vitro culture and implications for immunotherapy. Gynecol Oncol 65: 391-398, 1997.

20. Okada K, Yasumura S, Muller-Fleckenstein I, et al: Interactions between autologous $\mathrm{CD}^{+}$and $\mathrm{CD} 8^{+} \mathrm{T}$ lymphocytes and human squamous cell carcinoma of the head and neck. Cell Immunol 177: 35-48, 1997.
21. Marrogi AJ, Munshi A, Merogi AJ, et al: Study of tumor infiltrating lymphocytes and transforming growth factor beta as prognostic factors in breast carcinoma. Int J Cancer 74: 492-501, 1997.

22. Reichert TE, Strauss L, Wagner EM, Gooding W and Whiteside TL: Signaling abnormalities, apoptosis, and reduced proliferation of circulating and tumor infiltrating lymphocytes in patients with oral carcinoma. Clin Cancer Res 8: 3137-3145, 2002.

23. Ku TKS, Nguyen DC, Karaman M, Gill P, Hacia JG and Crowe DL: Loss of p53 expression correlates with metastatic phenotype and transcriptional profile in a new mouse model of head and neck cancer. Mol Cancer Res 5: 351-362, 2007.

24. Nguyen DC, Parsa B, Close A, Magnusson B, Sinha U and Crowe DL: Overexpression of cell cycle regulatory proteins correlates with advanced tumor stage in head and neck squamous cell carcinomas. Int J Oncol 22: 1285-1290, 2003

25. Andersen MH, Schrama D, Straten PT and Becker JC: Cytotoxic T cells. J Invest Dermatol 126: 32-41, 2006.

26. Gasser S and Raulet DH: The DNA damage response arouses the immune system. Cancer Res 66: 3959-3962, 2006.

27. Katou F, Ohtani H, Watanabe Y, Nakayama T, Yoshie O and Hashimoto K: Differing phenotypes between intraepithelial and stromal lymphocytes in early stage tongue cancer. Cancer Res 67: 11195-11201, 2007.

28. Willimsky G and Blankenstein T: Sporadic immunogenic tumors avoid destruction by inducing $\mathrm{T}$ cell tolerance. Nature 437 : 141-146, 2005.

29. Kim R, Emi M, Tanabe K and Arihiro K: Tumor driven evolution of immunosuppressive networks during malignant progression. Cancer Res 66: 5527-5536, 2006.

30. Koebel CM, Vermi W, Swann JB, et al: Adaptive immunity maintains occult cancer in an equilibrium state. Nature 450 : 903-908, 2007.

31. Renkvist N, Castelli C, Robbins PF and Parmiani G: A listing of human tumor antigens recognized by $\mathrm{T}$ cells. Cancer Immunol Immunother 50: 3-15, 2001.

32. Ciernik IF, Berzofsky JA and Carbone DP: Human lung cancer cells endogenously expressing mutant p53 process and present the mutant epitope and are lysed by mutant specific cytotoxic T lymphocytes. Clin Cancer Res 2: 877-882, 1996.

33. Castelli C, Rivoltini L, Andreola G, Carrabba M, Renkvist N and Parmiani G: $\mathrm{T}$ cell recognition of melanoma associated antigens. J Cell Physiol 182: 323-331, 2000.

34. Vonderheide RH, Hahn WC, Schultze JL and Nadler LM: The telomerase catalytic subunit is a widely expressed tumor associated antigen recognized by cytotoxic $\mathrm{T}$ lymphocytes. Immunity 10: 673-679, 1999. 